\title{
Serum uric acid level and its association with metabolic syndrome and carotid atherosclerosis in patients with type 2 diabetes
}

Qin Li ${ }^{1}$, Zhen Yang ${ }^{2 *}$, Bin Lu', Jie Wen ${ }^{1}$, Zi Ye ${ }^{1}$, Lili Chen ${ }^{1}$, Min He ${ }^{1}$, Xiaoming Tao ${ }^{1}$, Weiwei Zhang ${ }^{1}$, Ying Huang ${ }^{1}$, Zhaoyun Zhang ${ }^{1}$, Shen $\mathrm{Qu}^{2}$ and Renming $\mathrm{Hu}^{1 *}$

\begin{abstract}
Objective: We aimed to investigate whether elevated serum uric acid concentrations are associated with higher risk of metabolic syndrome (MetS) and carotid atherosclerosis in patients with type 2 diabetes.

Methods: We conducted a population-based cross-sectional survey in Shanghai, with a total of 395 men and 631 women age 41 to 92 years. The carotid artery intima-media thickness (IMT) and carotid atherosclerotic plaques (PLQ) were measured by B-mode ultrasound. MetS was defined according to the updated National Cholesterol Education Program Adult Treatment Panel III criteria for Asian Americans.

Results: Uric acid levels were negatively associated with duration of diabetes, fasting plasma glucose, glycohemoglobin, eGFR, HDL-cholesterol (all P < 0.001) and positively with BMI, CRP, waist circumference, triglycerides, systolic blood pressure, ACR, HOMA-IR and IMT (all P < 0.05). In the highest quartile of uric acid levels, the risks were substantially higher for MetS [odds ratio 3.97, (95\% confidence interval 2.58-6.13)] $(P<0.001$ for trend) and PLQ [odds ratio 2.71 (95\% confidence interval 1.62-4.47)] ( $p=0.013$ for trend) compared with that in the lowest quartile of uric acid levels after multiple adjustment. These associations remained significant after further adjustment for potential confounders.
\end{abstract}

Conclusions: Serum uric acid level is associated with MetS and is an independent risk factor for carotid atherosclerosis in patients with type 2 diabetes.

Keywords: uric acid, metabolic syndrome, intima-media thickness, atherosclerosis

\section{Introduction}

Metabolic syndrome (MetS), a clustering of cardiovascular risk factors such as insulin resistance, hypertension, glucose intolerance, hypertriglyceridemia, and low highdensity lipoprotein (HDL) cholesterol levels, is a major worldwide public health problem[1]. The prevalence of MetS is increasing in China because of the westernization of the lifestyle, such as a high-fat and high-calorie diet and less physical activity. A recent epidemiological study indicated that the prevalence of MetS among

\footnotetext{
* Correspondence: yangzhen1020@hotmail.com; renminghu@fudan.edu.cn 'Institute of endocrinology and diabetology, Huashan hospital, Shanghai medical college, Fudan University. 12 Middle Wulumuqi Road, Shanghai, China

${ }^{2}$ Department of endocrinology, Shanghai tenth people's hospital, School of medicine, Tongji University. 301 Middle Yanchang Road, Shanghai, China Full list of author information is available at the end of the article
}

Chinese men and women aged 35 to 64 years was $9.8 \%$ and $17.8 \%$, respectively[2].

Uric acid is the end product of purine metabolism in humans. Excess serum accumulation can lead to various diseases, and most notably uric acid is causally involved in the pathogenesis of gouty arthritis [3-5]. Recent studies have suggested that hyperuricemia is a risk factor for cardiovascular disease (CVD) in the general population [6-8]. However, despite the clinical and epidemiological evidence, some authorities have considered that the association was confounded by other well-established risk factors for CVD [3,9].

Recently, growing evidence demonstrates that uric acid may have a key role in the pathogenesis of MetS [10]. Recent studies in animals models report that uric acid may play a causal role in the development of MetS 
and decreasing uric acid levels can prevent or reverse features of the MetS [11].

Although previous studies have analyzed the independence of the relationship between uric acid and MetS and carotid atherosclerosis, thus far, few have examined in type 2 diabetes and there is a scarcity of data evaluating the effect of MetS on the association between serum uric acid and carotid atherosclerosis. In this study, we aimed to investigate the association between the serum uric acid and MetS and carotid atherosclerosis in patients with type 2 diabetes.

\section{Methods}

\section{Study subjects}

A cross-sectional study to evaluate the prevalence of diabetic complications in Chinese patients aged over 30 and diagnosed with type 2 diabetes was planned in downtown Shanghai. The details of the study design have been described previously[12]. In brief, the study was conducted from February to July 2004. 20 residential areas administered by 20 residents' committees were sampled randomly in the central area of Shanghai. Questionnaires to identify diabetes history were sent to every household in the 20 residential areas and collected by primary care clinicians and endocrinologists. A total of 1120 Chinese patients diagnosed with type 2 diabetes were identified via the questionnaire. Finally, 1039 Chinese patients diagnosed with type 2 diabetes were enrolled in our study. After excluding those who did not have serum uric acid data $(\mathrm{n}=13)$, 1026 individuals (395 men and 631 women) were eligible for the present analysis. The study was approved by the Institution Review Board of the Huashan Hospital, and written informed consents were obtained from all participants.

\section{Laboratory measurements}

Overnight fasting blood samples were collected in tubes containing liquid EDTA, centrifuged at $4^{\circ} \mathrm{C}$, and stored at $-80^{\circ} \mathrm{C}$ until analysis. The measurements of total cholesterol, HDL-cholesterol, low-density lipoprotein (LDL) cholesterol, triglycerides, glucose, insulin, creatinine, and C-reactive protein (CRP) were previously described $[12,13]$. Insulin resistance was estimated using homeostasis model assessment index-insulin resistance (HOMA-IR) [14]. A sterile, random-spot urine sample was used to measure the albumin/creatinine ratio (ACR). We used the modification of diet in renal disease (MDRD) equation re-calibrated for Chinese to estimate eGFR expressed in $\mathrm{ml} / \mathrm{min} / 1.73 \mathrm{~m}^{2}$ : eGFR $=186 \times[\mathrm{SCR}$ $\times 0.011]^{-1.154} \times[\text { age }]^{-0.203} \times[0.742$ if female $] \times 1.233$, where SCR is serum creatinine expressed as $\mu \mathrm{mol} / \mathrm{l}$ and 1.233 is the coefficient for Chinese.

\section{Carotid ultrasonography}

Carotid ultrasonography was performed using an Acuson Sequoia 512. Trained and certified sonographers conducted the examination. The ultrasound scanning protocol in our study was modified in terms of procedures used in previous studies [15-17]. Computerassisted edge-detection software was not used for measurement of carotid intima-media thickness (IMT). A lateral view of bilateral images of common carotid arteries $(1 \mathrm{~cm}$ proximal to the dilatation of the carotid bulb), carotid bulb (identified by the loss of the parallel wall present in the common carotid artery) and internal carotid artery $(1 \mathrm{~cm}$ distal to the tip of the flow divider that separates the external and internal carotid arteries) was obtained. Sonographers recorded the images and completed ultrasound readings. IMT is the distance between the lumen-intima interface and the mediaadventitia interface [18]. Common carotid artery IMT was defined as the mean of the maximum IMT in both right and left sides of common carotid artery.

The plaque of carotid artery (common carotid artery, carotid bulb and internal carotid artery) is defined as a localized protrusion of the internal part of the vessel wall into the lumen of $50 \%$ of the surrounding IMT value. Plaque presence was defined as $\geq 1$ plaque in any of the carotid arteries [19].

\section{Definition of MetS}

The MetS was defined based on the updated National Cholesterol Education Program Adult Treatment Panel III criteria for Asian-Americans[20]: (1) as presenting at least three of the following components: 1) waist circumferences $90 \mathrm{~cm}$ or greater in men or $80 \mathrm{~cm}$ or greater in women; 2) triglycerides $1.7 \mathrm{mmol} /$ liter or greater; 3) HDL cholesterol less than $1.03 \mathrm{mmol} / \mathrm{liter}$ in men or less than $1.30 \mathrm{mmol} /$ liter in women; 4) blood pressure 130/85 mm Hg or greater or current use of antihypertensive medications; or 5) fasting plasma glucose (FPG) $5.6 \mathrm{mmol} /$ liter or greater or previously diagnosed type 2 diabetes or on oral antidiabetic agents or insulin.

\section{Statistical analysis}

Normally distributed data were expressed as means \pm $\mathrm{SD}$, whereas variables with a skewed distribution were reported as median (interquartile range) and log transformed to approximate normality before analysis. Categorical variables were represented by frequency and percentage. Analysis of covariance for continuous variables and multivariate logistic regression analysis for categorical variables were applied for the comparison according to uric acid quartiles. Analysis of covariance was used to compare uric acid levels between genders. 
Correlation coefficients between uric acid and metabolic features were calculated by partial correlation analysis on ranks (Spearman correlation). Serum uric acid levels were depicted according to the number of MetS components using linear regression model. Multivariate logistic regression models were used to estimate the odds ratios (ORs) for MetS. Potential confounding variables including age, gender, smoking, alcohol drinking, self-reported CVD, family history of diabetes, eGFR, $\mathrm{HbA}_{1 \mathrm{C}}, \mathrm{CRP}$, HOMA-IR and body mass index (BMI) were controlled in the regression models. Data management and statistical analysis were performed using SPSS version 13.0 for Windows (SPSS, Chicago, IL, USA). P $<0.05$ was considered statistically significant.

\section{Results}

Characteristics of participants according to serum uric acid quartiles

We identified 1026 patients with type 2 diabetes with a mean age of $65.57 \pm 11.70$ years. At the time of uric acid determination, history of hypertension was documented in 761 patients (74.2\%) and CVD in 200 patients (19.5\%). Baseline demographic and medical characteristics for both genders combined and divided by uric acid quartiles are illustrated in Table 1 . When analyzed by quartiles of uric acid levels, the patients with higher uric acid were more likely to be male and smokers (both $\mathrm{P}<$ $0.05)$. With respect to metabolic parameters, the patients in the higher uric acid quartiles exhibited higher levels of systolic blood pressure, BMI, waist circumference, HOMA-IR, CRP, IMT, creatinine, ACR and triglycerides (all $\mathrm{P}<0.05$ ). In contrast, the patients with higher uric acid levels displayed shorter duration of diabetes and lower levels of FPG, $\mathrm{HbA}_{1 \mathrm{C}}$, eGFR and HDL cholesterol (all $\mathrm{P}<0.001$ ).

\section{Association between serum uric acid and MetS}

Partial correlation analysis demonstrated strong correlation between uric acid and BMI, waist circumference, HOMA-IR, CRP, TG and HDL cholesterol among various metabolic features (Table 2).

Remarkably, serum uric acid levels increased gradually with increasing number of MetS components (Figure 1). The mean values (SE) of uric acid concentrations significantly increased for those with one to five components were 246.67 (7.89), 268.31 (4.37), 277.91 (4.28), 303.35 (4.56), and $330.35(6.70) \mu \mathrm{mol} / \mathrm{L}$, respectively, after adjustment for age, gender, creatinine, alcohol drinking, smoking, duration of diabetes, self-reported CVD and family history of diabetes.

As presented in Table 3, the ORs for MetS were higher with increasing uric acid quartiles $(\mathrm{P}<0.001$ for trend). In the highest uric acid quartile, the ORs were 3.97 [95\% confidence interval (CI) 2.58-6.13] for MetS after adjusting for age, gender, creatinine, alcohol drinking, smoking, duration of diabetes and family history of diabetes.

\section{Association between serum uric acid and carotid atherosclerosis}

Partial correlation analysis demonstrated a significant correlation between uric acid and IMT ( $\mathrm{p}=0.02$ ) (Table 2).

As presented in Table 3, the ORs for carotid atherosclerotic plaques (PLQ) were higher with increasing uric acid quartiles ( $\mathrm{p}=0.013$ for trend). In the highest uric acid quartile, the ORs were 2.71 [95\% confidence interval (CI) 1.62-4.47] for PLQ after adjusting for age, gender, eGFR, alcohol drinking, smoking, duration of diabetes and family history of diabetes.

\section{Effect of MetS on the association between serum uric acid and PLQ}

After adjusting for age, gender, eGFR, alcohol drinking, smoking, duration of diabetes and family history of diabetes, MetS was associated with the PLQ with an odds ratio of 2.24 (95\% CI 1.34-3.57, $\mathrm{P}<0.001$ ). Thus, we next investigated the association between serum uric acid and PLQ according to the status of MetS. After adjusting for age, gender, $\mathrm{HbA}_{1 \mathrm{C}}, \mathrm{BMI}$, systolic blood pressure, diastolic blood pressure, FPG, alcohol drinking, smoking, duration of diabetes and family history of diabetes, in the highest uric acid quartile, the ORs were 1.85 (95\% CI 1.14-2.83, $\mathrm{p}=0.023$ ) for carotid plaque in subjects without MetS and 3.07 (95\% CI 1.25-6.42) in subjects with MetS.

\section{Serum uric acid level and glucose control}

Hyperglycaemia as reflected by mean $\mathrm{HbA}_{1 \mathrm{C}}$ levels was associated with lower serum uric acid levels. As summarized in Table 1, the patients with higher uric acid levels displayed lower $\mathrm{HbA}_{1 \mathrm{C}}$ and fasting glucose levels (both $\mathrm{P}<0.001$ ). $\mathrm{HbA}_{1 \mathrm{C}}$ and FPG were negatively correlated with serum uric acid (Table 2). Moreover, we also observed that serum uric acid levels were negatively related to the duration of diabetes $(\mathrm{P}<0.001)$.

\section{Discussion}

In the present study, we found that serum uric acid levels showed association with the risk of MetS and carotid atherosclerosis from the cross-sectional data in type 2 diabetes. Moreover, these associations are independent of lifestyle factors, eGFR, duration of diabetes, family history of diabetes and remarkably, CRP, $\mathrm{HbA}_{1 \mathrm{C}}$, HOMA-IR and BMI.

Possible associations between uric acid and MetS were evaluated in some studies [10,21]. We also observed that the ORs were substantially higher for MetS [OR 3.97, (95\% confidence interval 2.58-6.13)] $(\mathrm{P}<0.001$ for 
Table 1 Characteristics of study participants according to uric acid quartiles ${ }^{\mathrm{a}}$

\begin{tabular}{|c|c|c|c|c|c|}
\hline Characteristics & $\begin{array}{c}\text { Q1 } \\
(n=256) \\
<230\end{array}$ & $\begin{array}{c}\text { Q2 } \\
(\mathrm{n}=257) \\
231-280\end{array}$ & $\begin{array}{c}\text { Q3 } \\
(n=256) \\
281-330\end{array}$ & $\begin{array}{c}\text { Q4 } \\
(n=257) \\
\geq 331\end{array}$ & $P$ value \\
\hline MetS (\%) & 20.3 & 35.8 & 54.3 & 84.8 & $<0.001$ \\
\hline Uric acid $(\mu \mathrm{mol} / \mathrm{L})$ & $200(190-220)$ & $260(250-270)$ & $310(300-320)$ & $390(350-430)$ & $<0.001$ \\
\hline Age $(y r)^{b}$ & $65.28 \pm 11.27$ & $66.57 \pm 10.28$ & $65.09 \pm 12.35$ & $67.52 \pm 11.84$ & 0.065 \\
\hline Male & $73(28.29)$ & 91 (30.33) & $92(41.81)$ & $139(56.05)$ & $<0.001$ \\
\hline Smoking (yes) & $47(18.22)$ & $47(15.67)$ & $52(23.64)$ & $63(25.40)$ & 0.017 \\
\hline Alcohol (yes) & $27(10.47)$ & $27(9.00)$ & $31(15.50)$ & $38(15.32)$ & 0.084 \\
\hline Duration of diabetes (yr) & $9.72 \pm 7.84$ & $7.74 \pm 7.04$ & $7.24 \pm 6.69$ & $6.75 \pm 6.56$ & $<0.001$ \\
\hline Self-reported CVD ${ }^{c}$ & 49 (18.99) & $62(20.67)$ & $49(22.27)$ & $59(23.79)$ & 0.587 \\
\hline Family history of diabetes $^{d}$ & $112(43.41)$ & $119(39.67)$ & $74(33.64)$ & $84(33.87)$ & 0.068 \\
\hline $\mathrm{SBP}(\mathrm{mmHg})$ & $138.44 \pm 20.68$ & $139.92 \pm 19.82$ & $140.86 \pm 22.00$ & $142.31 \pm 20.05$ & $<0.001$ \\
\hline $\mathrm{DBP}(\mathrm{mmHg})$ & $80.71 \pm 10.54$ & $82.57 \pm 10.92$ & $82.14 \pm 12.18$ & $82.38 \pm 10.69$ & 0.207 \\
\hline $\mathrm{BMI}\left(\mathrm{kg} / \mathrm{m}^{2}\right)$ & $24.21 \pm 3.39$ & $24.91 \pm 3.23$ & $25.40 \pm 3.47$ & $25.97 \pm 3.39$ & $<0.001$ \\
\hline Waist circumference $(\mathrm{cm})$ & $81.74 \pm 9.68$ & $83.62 \pm 8.50$ & $86.05 \pm 9.32$ & $87.67 \pm 8.88$ & $<0.001$ \\
\hline Waist to hip ratio & $0.86 \pm 0.08$ & $0.87 \pm 0.06$ & $0.88 \pm 0.06$ & $0.89 \pm 0.06$ & 0.176 \\
\hline Glucose (mmol/L) & $9.25(7.20-12.30)$ & $7.70(6.40-9.60)$ & $7.80(6.60-9.70)$ & $7.30(6.23-8.80)$ & $<0.001$ \\
\hline Insulin $(\mu \mathrm{U} / \mathrm{mL})^{\mathrm{e}}$ & $9.93(5.85-15.06)$ & $11.06(6.93-17.26)$ & $12.40(7.93-18.24)$ & $13.19(8.80-20.13)$ & 0.143 \\
\hline HOMA-IR & $3.91(2.28-6.70)$ & $3.98(2.36-7.21)$ & $4.40(2.62-7.38))$ & $4.44(2.63-6.55$ & 0.028 \\
\hline CRP & $1.92(0.95-4.35)$ & $1.98(0.71-4.92)$ & $2.33(0.73-5.24)$ & $3.59(0.81-8.87)$ & $<0.001$ \\
\hline IMT (mm) & $0.82 \pm 0.21$ & $0.85 \pm 0.22$ & $0.86 \pm 0.26$ & $0.93 \pm 0.29$ & 0.016 \\
\hline $\mathrm{HbA}_{1 \mathrm{C}}(\%)$ & $7.91 \pm 1.94$ & $7.10 \pm 1.59$ & $6.96 \pm 1.42$ & $6.93 \pm 1.36$ & $<0.001$ \\
\hline Creatinine ( $\mu \mathrm{mol} / \mathrm{L})$ & $60.67 \pm 13.14$ & $65.22 \pm 19.63$ & $67.35 \pm 15.50$ & $81.09 \pm 28.75$ & $<0.001$ \\
\hline eGFR $\left(\mathrm{ml} / \mathrm{min} / 1.73^{2}\right)$ & $132.5 \pm 25.5$ & $125.2 \pm 25.4$ & $122.5 \pm 26.7$ & $108.3 \pm 31.2$ & $<0.001$ \\
\hline ACR (mg/mmol) & $2.45(1.25-6.04)$ & $2.80(1.35-9.59)$ & $3.02(1.54-8.11)$ & $3.52(1.74-8.94)$ & 0.022 \\
\hline Triglycerides $(\mathrm{mmol} / \mathrm{L})^{\mathrm{e}}$ & $1.41(0.97-1.91)$ & $1.56(1.09-2.23)$ & $1.67(1.17-2.41)$ & $1.84(1.28-2.64)$ & $<0.001$ \\
\hline Total cholerterol (mmol/L) & $5.32 \pm 0.07$ & $5.37 \pm 0.07$ & $5.44 \pm 0.08$ & $5.33 \pm 0.07$ & 0.623 \\
\hline LDL cholesterol (mmol/L) & $2.99 \pm 0.90$ & $3.04 \pm 0.84$ & $3.11 \pm 0.86$ & $3.07 \pm 0.85$ & 0.189 \\
\hline HDL cholesterol (mmol/L) & $1.47 \pm 0.40$ & $1.37 \pm 0.36$ & $1.26 \pm 0.35$ & $1.14 \pm 0.29$ & $<0.001$ \\
\hline
\end{tabular}

SBP, systolic blood pressure; DBP, diastolic blood pressure.; IMT, intima-media thickness

${ }^{\mathrm{a}}$ Data are means SD, median (interquartile range), or number (percent); P value was calculated after adjustment for age, gender.

${ }^{\mathrm{b}}$ Not adjusted for itself.

'Self-reported CVD including stroke and coronary heart disease.

${ }^{d}$ Parents or siblings had a history of diabetes.

'These variables were log transformed before analysis.

trend) and PLQ [OR 2.71 (95\% confidence interval 1.624.47)] ( $p=0.013$ for trend) in the highest serum uric acid quartile compared with those in the lowest quartile. In addition, we observed the serum uric acid concentration increased monotomically with the number of MetS components $(\mathrm{P}<0.001$ for trend). However, the underlying mechanisms of these associations are still largely unknown although much research has been done in this area. Recent studies indicate that hyperuricemia may be partially responsible for the proinflammatory endocrine imbalance in the vascular smooth muscle cells and adipose tissue, which is an underlying mechanism of the low-grade inflammation and insulin resistance in subjects with the CVD and MetS [22,23]. Lowering uric acid in mice by allopurinol could improve the proinflammatory endocrine imbalance in the adipose tissue by reducing production of monocyte chemoattractant protein-1 (MCP-1) and increasing production of adiponectin. In addition, lowering uric acid in obese mice decreased macrophage infiltration in the adipose tissue and reduced insulin resistance [21]. Moreover, uric acid has also been shown to induce production of interleukin $1 \beta$, interleukin 6 , tumor necrosis factor $\alpha$ in human mononuclear cells, and CRP in cultured human vascular cells[24], all these have been suggested that uric acid may have some interaction with other inflammatory cytokines. In line with views, we observed significant correlation between serum uric acid and CRP levels even after adjustment for other potential confounders ( $\mathrm{r}$ $=0.08, \mathrm{p}=0.01$ ). Furthermore, we also observed that CRP increased with serum uric acid quartiles in this study $(\mathrm{P}<0.001)$, which indicate that uric acid is 
Table 2 Correlation between serum uric acid and other

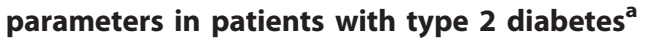

\begin{tabular}{lcc}
\hline Variable & Correlation coefficient & P value \\
\hline Age & 0.04 & 0.19 \\
BMI & 0.15 & $<0.001$ \\
Duration of diabetes & -0.14 & $<0.001$ \\
Waist circumference & 0.21 & $<0.001$ \\
Waist to hip ratio & 0.08 & 0.01 \\
SBP & 0.04 & 0.23 \\
DBP & 0.02 & 0.48 \\
CRP & 0.08 & 0.01 \\
Glucose & -0.26 & $<0.001$ \\
Insulin & 0.11 & $<0.001$ \\
HbA 1 C & -0.24 & $<0.001$ \\
HOMA-IR & 0.07 & 0.02 \\
IMT & 0.07 & 0.02 \\
Creatinine & 0.33 & $<0.001$ \\
eGFR & -0.36 & $<0.001$ \\
ACR & 0.11 & $<0.001$ \\
Triglycerides & -0.04 & 0.20 \\
Total cholesterol & 0.20 & $<0.001$ \\
LDL cholesterol & 0.01 & 0.80 \\
HDL cholesterol & -0.33 & $<0.001$ \\
\hline
\end{tabular}

IMT, intima-media thickness; SBP, systolic blood pressure; DBP, diastolic blood pressure;

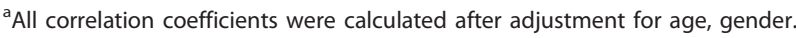

correlated with low-grade systemic inflammatory response and could potentially modulate chronic inflammatory processes[25]. It is well-known that subclinical inflammation is a risk factor not only for MetS but also for diabetes complications such as CVD and stroke

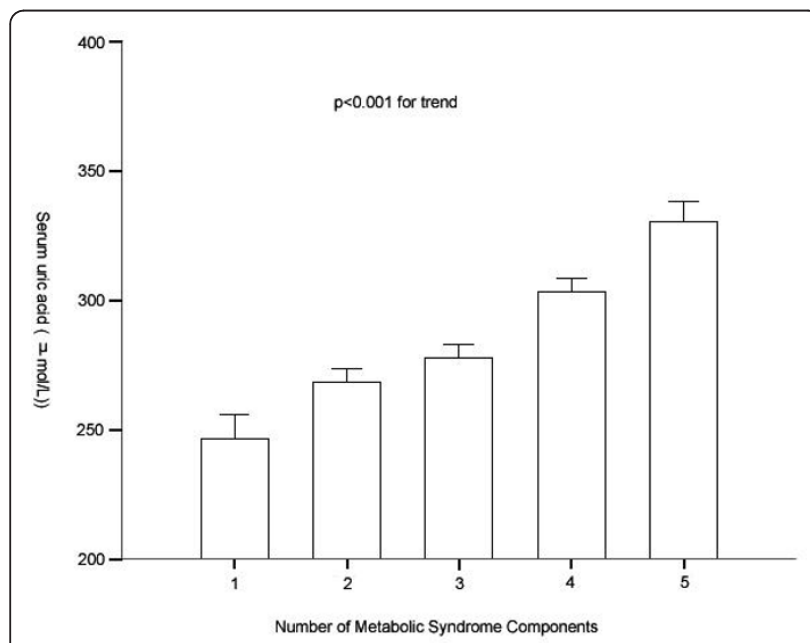

Figure 1 Plasma uric acid levels according to the number of MetS components. Data are shown as means \pm SE after adjustment for age, gender, eGFR, alcohol drinking, smoking, duration of diabetes, self-reported CVD, and family history of diabetes; $\mathrm{P}<0.001$ for trend.
$[26,27]$. Taken together, these important and intriguing results suggest that uric acid may play a causal role in the pathogenesis of atherosclerosis and the MetS partially by inflammatory pathway.

Given the serum uric acid levels were significantly associated with atherosclerosis and the MetS[28,29], it is plausible to consider uric acid as a promising candidate for risk assessment and a potential intervention target for CVD and MetS. Interestingly, in line with our hypothesis, some studies have demonstrated that reducing uric acid by treatment with allopurinol significantly improved endothelial function, peripheral vasodilator capacity and blood flow both locally and systemically, which represent a new potential therapeutic approach [30-32]. Certainly, prospective studies with solid clinical end points are urgently needed to clarify whether a high uric acid level plays a causal role in the development of CVD and MetS.

One of the interesting findings of the present study is the inverse association of serum uric acid with diabetic parameters (duration of diabetes, $\mathrm{HbA}_{1 \mathrm{C}}$, FPG). The inverse association of serum uric acid with diabetic parameters is counterintuitive. Historically, the elevated level of uric acid observed in the MetS has been attributed to hyperinsulinemia, since hyperinsulinemia is known to be associated with impaired renal uric acid excretion and hyperuricemia in these patients are likely to be due to retention of uric acid[33]. But here in our current study, the type 2 diabetes patients with a higher $\mathrm{HbA}_{1 \mathrm{C}}$, fasting glucose and a longer duration of diabetes are likely to be accompanied by worsening function of beta cells to secrete adequate amounts of insulin. And the inverse relationship between serum uric acid and diabetic parameters (duration of diabetes, $\mathrm{HbA}_{1 \mathrm{C}}, \mathrm{FPG}$ ) observed in these diabetic subjects may probably be caused by the increased renal excretion of uric acid in the presence of hyperglycaemia. It is well-known that with the increasing of the duration of diabetes, which is accompanied by worsening of the function of beta cells and deterioration of glycemic control, the rate of renal filtration in patients with diabetes increases gradually. The hyperfiltration state which caused by hyperglycaemia promotes the excretion of uric acid, which partly explain the existence of the inverse relationship between serum uric acid and diabetic parameters. Patients with long-term hyperfiltration state may eventually develop into diabetic nephropathy. Consistent with our hypothesis, we observed that ACR increased and eGFR decreased with serum uric acid quartiles in this study ( $\mathrm{p}$ values were 0.022 and $3.7 \times 10^{-5}$, respectively).

As a cross-sectional study, there are several limitations. The mechanisms underlying these associations are 
Table 3 Adjusted ORs and $95 \%$ Cls for MetS and carotid plaque according to uric acid quartiles

\begin{tabular}{|c|c|c|c|c|c|}
\hline & \multicolumn{4}{|c|}{ ORs $(95 \% \mathrm{Cl})$} & \multirow[t]{2}{*}{$P$ value for trend } \\
\hline & Q1 & Q2 & Q3 & Q4 & \\
\hline \multicolumn{6}{|l|}{$\overline{\text { MetS }}$} \\
\hline Model $1^{\mathrm{a}}$ & 1.0 & $1.42(0.99-2.04)$ & $2.27(1.51-3.42)$ & $3.97(2.58-6.13)$ & $<0.001$ \\
\hline Model $2^{b}$ & 1.0 & $1.37(0.95-1.98)$ & $2.21(1.44-3.39)$ & $3.65(2.33-5.71)$ & $<0.001$ \\
\hline Model $3^{c}$ & 1.0 & $1.33(0.88-2.07)$ & $2.27(1.36-3.71)$ & $3.77(2.26-6.29)$ & $<0.001$ \\
\hline Model $4^{d}$ & 1.0 & $1.22(0.82-1.83)$ & $1.93(1.22-3.03)$ & $3.26(2.03-5.25)$ & $<0.001$ \\
\hline \multicolumn{6}{|l|}{ carotid plaque } \\
\hline Model $1^{*}$ & 1.0 & $1.33(0.88-1.89)$ & $1.89(1.16-2.85)$ & $2.71(1.62-4.47)$ & 0.013 \\
\hline Model $2^{\dagger}$ & 1.0 & $1.29(0.82-1.81)$ & $1.82(1.13-2.77)$ & $2.53(1.49-4.13)$ & 0.019 \\
\hline Model $3^{\ddagger}$ & 1.0 & $1.22(0.81-1.85)$ & $1.68(1.12-2.58)$ & $2.42(1.40-3.75)$ & 0.020 \\
\hline Model $4^{\xi}$ & 1.0 & $1.18(0.80-1.76)$ & $1.52(1.04-2.50)$ & $2.21(1.32-3.49)$ & 0.036 \\
\hline
\end{tabular}

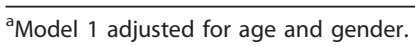

${ }^{\mathrm{b}}$ Model 2 further adjusted for alcohol drinking, smoking, duration of diabetes, and family history of diabetes.

${ }^{c}$ Model 3 further adjusted for eGFR, CRP, $\mathrm{HbA}_{1 \mathrm{C}}$ and HOMA-IR.

${ }^{\mathrm{d}}$ Model 4 further adjusted for BMI.

*Model 1 adjusted for age and gender.

${ }^{\dagger}$ Model 2 further adjusted for alcohol drinking, smoking, duration of diabetes, and family history of diabetes.

${ }^{\ddagger}$ Model 3 further adjusted for eGFR, CRP, $\mathrm{HbA}_{1 \mathrm{C}}$ and HOMA-IR.

${ }^{\xi}$ Model 4 further adjusted for BMI and MetS.

still to be explored. The present findings are inherently limited in the ability to eliminate causal relationships between uric acid and MetS. Although most potential confounders were carefully controlled, since some of the study patients had several risk factors including hypertension and dyslipidemia, we could not eliminate the possible effect of underlying diseases and medications used for these diseases on the present findings. Further prospective population-based trials are needed to investigate the mechanisms in order to answer these questions.

In conclusion, our data indicate that serum uric acid levels are significantly associated with MetS and carotid atherosclerosis in patients with type 2 diabetes, even after adjustment for other potential confounders. Furthermore, our findings suggest that the observed association between uric acid and carotid atherosclerosis may be attributed to metabolic syndrome-dependent and -independent mechanisms. Prospective studies are required to assess the time course and causal relevance of serum uric acid in the development of MetS and carotid atherosclerosis in patients with type 2 diabetes.

\section{Conflict of interests statement}

The authors declare that they have no competing interests.

\footnotetext{
Acknowledgements

This work was supported by the National Natural Science Foundation of China (81000344, 81070238, 81030014), Shanghai Education Committee Foundation (11ZZ33), Shanghai Pujiang Foundation(PJ1408400), Shanghai Science and Technology Commission (08JC1403200, 09DZ1950200), the National High Technology Research and Development Program ("863"
}

Program) of China (2009AA022704), China Postdoctoral Science Foundation (20080440078), and Shanghai Postdoctoral Scientific Program (09R21411600).

\section{Author details}

${ }^{1}$ Institute of endocrinology and diabetology, Huashan hospital, Shanghai medical college, Fudan University. 12 Middle Wulumuqi Road, Shanghai, China. 'Department of endocrinology, Shanghai tenth people's hospital, School of medicine, Tongji University. 301 Middle Yanchang Road, Shanghai, China.

\section{Authors' contributions}

ZY and RH designed the study; QL, ZY, LC, XT and WZ participated in acquisition of data; JW, ZZ, BL and SQ researched and evaluated the literature; ZY undertook the statistical analysis and QL wrote the first draft of the manuscript. All authors have approved the final manuscript for publication.

Received: 23 July 2011 Accepted: 4 August 2011

Published: 4 August 2011

\section{References}

1. Yamaoka-Tojo M, Tojo T, Takahira N, Matsunaga A, Aoyama N, Masuda T, Izumi T: Elevated circulating levels of an incretin hormone, glucagon-like peptide-1, are associated with metabolic components in high-risk patients with cardiovascular disease. Cardiovascular Diabetology 2010, 9(17)

2. Gu DF, Reynolds K, Wu XG, Chen F, Duan XF, Reynolds RF, Whelton PK, He J, Inter ACG: Prevalence of the metabolic syndrome and overweight among adults in China. Lancet 2005, 365(9468):1398-1405.

3. Moriarity JT, Folsom AR, Iribarren C, Nieto FJ, Rosamond WD: Serum Uric Acid and Risk of Coronary Heart Disease: Atherosclerosis Risk in Communities (ARIC) Study. Annals of Epidemiology 2000, 10(3):136-143.

4. So A, Thorens B: Uric acid transport and disease. Journal of Clinical Investigation 2010, 120(6):1791-1799.

5. Koenig W, Meisinger C: Uric acid, type 2 diabetes, and cardiovascular diseases: Fueling the common soil hypothesis? Clinical Chemistry 2008, 54(2):231-233.

6. Meisinger C, Koenig W, Baumert J, Doring A: Uric acid levels are associated with all-cause and cardiovascular disease mortality independent of systemic inflammation in men from the general population - The MONICA/KORA cohort study. Arteriosclerosis Thrombosis and Vascular Biology 2008, 28(6):1186-1192. 
7. Verdecchia P, Schillaci G, Reboldi G, Santeusanio F, Porcellati C, Brunetti P: Relation between serum uric acid and risk of cardiovascular disease in essential hypertension the PIUMA study. Hypertension 2000, 36(6):1072-1078

8. Mankovsky B, Kurashvili R, Sadikot $\mathrm{S}$ : Is serum uric acid a risk factor for atherosclerotic cardiovascular disease?: A review of the clinical evidence. Part 1. Diabetes and Metabolic Syndrome: Clinical Research and Reviews 2010, 4(3):176-184.

9. Mazzali M, Hughes J, Kim YG, Jefferson JA, Kang DH, Gordon KL, Lan HY, Kivlighn S, Johnson RJ: Elevated uric acid increases blood pressure in the rat by a novel crystal-independent mechanism. Hypertension 2001, 38(5):1101-1106.

10. Ishizaka N, Ishizaka Y, Toda E-I, Nagai R, Yamakado M: Association Between Serum Uric Acid, Metabolic Syndrome, and Carotid Atherosclerosis in Japanese Individuals. Arterioscler Thromb Vasc Biol 2005, 25(5):1038-1044.

11. Nakagawa T, Hu H, Zharikov S, Tuttle KR, Short RA, Glushakova O, Ouyang X, Feig DI, Block ER, Herrera-Acosta J, Patel JM, Johnson RJ: A causal role for uric acid in fructose-induced metabolic syndrome. American Journal of Physiology - Renal Physiology 2006, 290(3):F625-F631.

12. Lu B, Wen J, Song XY, Dong $X H$, Yang $Y H$, Zhang $Z Y$, Zhao NQ, Ye HY, Mou B, Chen FL, Liu Y, Shen Y, Wang XC, Zhou LN, Li YM, Zhu XX, Hu RM: High prevalence of albuminuria in population-based patients diagnosed with type 2 diabetes in the Shanghai downtown. Diabetes Research and Clinical Practice 2007, 75(2):184-192.

13. Yang Z, Zhang ZY, Wen J, Wang XC, Lu B, Yang ZH, Zhang WW, Wang M, Feng XC, Ling C, Wu SH, Hu RM: Elevated Serum Chemokine CXC Ligand 5 Levels Are Associated with Hypercholesterolemia But Not a Worsening of Insulin Resistance in Chinese People. Journal of Clinical Endocrinology \& Metabolism 2010, 95(8):3926-3932

14. Matthews D, Hosker J, Rudenski A, Naylor B, Treacher D, Turner R: Homeostasis model assessment: insulin resistance and $\beta$-cell function from fasting plasma glucose and insulin concentrations in man. Diabetologia 1985, 28(7):412-419.

15. Epidemiology of Diabetes Interventions and Complications (EDIC) Research Group: Effect of intensive diabetes treatment on carotid artery wall thickness in the epidemiology of diabetes interventions and complications. Diabetes 1999, 48(2):383-390.

16. Dagostino RB, Burke G, Oleary D, Rewers M, Selby J, Savage PJ, Saad MF, Bergman RN, Howard G, Wagenknecht L, Haffner SM: Ethnic differences in carotid wall thickness - The Insulin Resistance Atherosclerosis Study. Stroke 1996, 27(10):1744-1749.

17. Wei M, Gonzalez C, Haffner SM, Oleary DH, Stern MP: Ultrasonographically assessed maximum carotid artery wall thickness in Mexico City residents and Mexican Americans living in San Antonio, Texas - Association with diabetes and cardiovascular risk factors. Arteriosclerosis Thrombosis and Vascular Biology 1996, 16(11):1388-1392.

18. Poli A, Tremoli E, Colombo A, Sirtori M, Pignoli P, Paoletti R: Ultrasonographic measurement of the common carotid artery wall thickness in hypercholesterolemic patients. A new model for the quantitation and follow-up of preclinical atherosclerosis in living human subjects. Atherosclerosis 1988, 70(3):253-261.

19. Lu B, Yang Y, Song X, Dong X, Zhang Z, Zhou L, Li Y, Zhao N, Zhu X, Hu R: An evaluation of the International Diabetes Federation definition of metabolic syndrome in Chinese patients older than 30 years and diagnosed with type 2 diabetes mellitus. Metabolism 2006, 55(8):1088-1096

20. Liu J, Grundy SM, Wang W, Smith SC, Vega GL, Wu Z, Zeng Z, Wang W, Zhao D: Ethnic-Specific Criteria for the Metabolic Syndrome. Diabetes Care 2006, 29(6):1414-1416.

21. Kim ES, Kwon HS, Ahn CW, Lim DJ, Shin JA, Lee SH, Cho JH, Yoon KH, Kang MI, Cha BY, Son HY: Serum uric acid level is associated with metabolic syndrome and microalbuminuria in Korean patients with type 2 diabetes mellitus. Journal of Diabetes and its Complications 2010.

22. Kanellis J, Watanabe S, Li JH, Kang DH, Li P, Nakagawa T, Wamsley A, Sheikh-Hamad D, Lan HY, Feng L, Johnson RJ: Uric Acid Stimulates Monocyte Chemoattractant Protein-1 Production in Vascular Smooth Muscle Cells Via Mitogen-Activated Protein Kinase and Cyclooxygenase2. Hypertension 2003, 41(6):1287-1293.

23. Baldwin W MS, Marek G, Wymer D, Pannu V, Baylis C, Johnson RJ, Sautin YY: Hyperuricemia as a Mediator of the Proinflammatory Endocrine
Imbalance in the Adipose Tissue in a Murine Model of the Metabolic Syndrome. Diabetes 2011, 60(4):1258-1269.

24. Kanellis J, Kang DH: Uric acid as a mediator of endothelial dysfunction, inflammation, and vascular disease. Seminars in Nephrology 2005, 25(1):39-42.

25. Tsai WC, Huang YY, Lin CC, Li WT, Lee CH, Chen JY, Chen JH: Uric acid is an independent predictor of arterial stiffness in hypertensive patients. Heart and Vessels 2009, 24(5):371-375.

26. Kawamoto R, Tabara Y, Kohara K, Miki T, Kusunoki T, Takayama S, Abe M, Katoh T, Ohtsuka N: High-sensitivity c-reactive protein and gammaglutamyl transferase levels are synergistically associated with metabolic syndrome in community-dwelling persons. Cardiovascular Diabetology 2010, 9.

27. Pu L, Xu XW, Zhang RY, Zhang Q, Zhang JS, Hu J, Yang ZK, Ding FH, Chen QJ, Lou S, Shen J, Fang DH, Shen WF: Value of serum glycated albumin and high-sensitivity C-reactive protein levels in the prediction of presence of coronary artery disease in patients with type 2 diabetes. Cardiovascular Diabetology 2006, 9.

28. Fukui M, Tanak M, Shiraishi E, Harusato I, Hosoda H, Asano M, Kadono M, Hasegawa G, Yoshikawa T, Nakamura N: Serum uric acid is associated with micro albuminuria and subclinical atherosclerosis in men with type 2 diabetes mellitus. Metabolism-Clinical and Experimental 2008, 57(5):625-629.

29. Kawamoto R, Tomita H, Oka Y, Ohtsuka N: Relationship between serum uric acid concentration, metabolic syndrome and carotid atherosclerosis. Internal Medicine 2006, 45(9):605-614.

30. Butler R, Morris AD, Belch JJF, Hill A, Struthers AD: Allopurinol normalizes endothelial dysfunction in type 2 diabetics with mild hypertension. Hypertension 2000, 35(3):746-751.

31. Farquharson CA, Butler R, Hill A, Belch JJF, Struthers AD: Allopurinol improves endothelial dysfunction in chronic heart failure. Circulation 2002, 106(2):221-226.

32. Doehner W, Schoene N, Rauchhaus M, Leyva-Leon F, Pavitt DV, Reaveley DA, Schuler G, Coats AJS, Anker SD, Hambrecht R: Effects of xanthine oxidase inhibition with allopurinol on endothelial function and peripheral blood flow in hyperuricemic patients with chronic heart failure - Results from 2 placebo-controlled studies. Circulation 2002, 105(22):2619-2624.

33. Facchini F, Chen Y-DI, Hollenbeck CB, Reaven GM: Relationship Between Resistance to Insulin-Mediated Glucose Uptake, Urinary Uric Acid Clearance, and Plasma Uric Acid Concentration. JAMA: The Journal of the American Medical Association 1991, 266(21):3008-3011.

doi:10.1186/1475-2840-10-72

Cite this article as: Li et al: Serum uric acid level and its association with metabolic syndrome and carotid atherosclerosis in patients with type 2 diabetes. Cardiovascular Diabetology 2011 10:72.

\section{Submit your next manuscript to BioMed Central and take full advantage of:}

- Convenient online submission

- Thorough peer review

- No space constraints or color figure charges

- Immediate publication on acceptance

- Inclusion in PubMed, CAS, Scopus and Google Scholar

- Research which is freely available for redistribution

Submit your manuscript at www.biomedcentral.com/submit
C Biomed Central 